\title{
Differentiation of Human Induced Pluripotent Stem Cells into a Keratinocyte Lineage
}

\author{
Igor Kogut ${ }^{1,2}$, Dennis R. Roop ${ }^{1,2}$, and Ganna Bilousova ${ }^{1,2,3}$ \\ ${ }^{1}$ Department of Dermatology, University of Colorado, Anschutz Medical Campus, Aurora, \\ Colorado, USA \\ ${ }^{2}$ Charles C. Gates Center for Regenerative Medicine and Stem Cell Biology, University of \\ Colorado, Anschutz Medical Campus, Aurora, Colorado, USA
}

\begin{abstract}
Direct reprogramming of somatic cells into induced pluripotent stem cells (iPSCs) provides an opportunity to develop novel personalized treatment options for numerous diseases and to advance current approaches for cell-based drug discoveries and disease modeling. The ability to differentiate iPSCs into relevant cell types is an important prerequisite for the successful development of iPSC-based treatment and modeling strategies. Here, we describe a protocol for the efficient differentiation of human iPSCs into functional keratinocytes. The protocol employs treating iPSCs with retinoic acid and bone-morphogenetic protein- 4 to induce differentiation toward a keratinocyte lineage, which is then followed by the growth of differentiated iPSCs on collagen type I- and collagen type IV-coated dishes to enrich for iPSC-derived keratinocytes.
\end{abstract}

\section{Keywords}

Induced pluripotent stem cells; iPSCs; Differentiation; Keratinocytes; Retinoic acid; Bonemorphogenetic protein-4

\section{Introduction}

The discovery that the ectopic expression of selected transcription factors can reprogram somatic cells into embryonic stem cell (ESC)-like cells, termed induced pluripotent stem cells (iPSCs), has opened up a new era in research and therapy (1-5). The iPSC technology addresses many obstacles associated with the use of ESCs, including ethical concerns, and allows for the generation of patient-specific pluripotent stem cells, which can be genetically corrected, differentiated into adult lineages, and returned to the same patient as an autograft (6-9). In addition to genetic disorders, the iPSC technology can be applicable to tissue regeneration, basic science research of human development, and disease modeling. However, before iPSC-based approaches are successfully implemented into the clinic,

\footnotetext{
${ }^{3}$ Correspondence: Ganna Bilousova, Charles C. Gates Center for Regenerative Medicine and Stem Cell Biology, University of Colorado, Anschutz Medical Campus, P.O. Box 6511, Mail Stop 8320, Aurora, CO 80045. Phone: 303-724-3957; Fax: 303-724-3051; Ganna.Bilousova@UCDenver.edu.
} 
efficient protocols for the differentiation of iPSCs into relevant cell types need to be developed.

In this chapter, we describe a protocol for the efficient differentiation of human iPSCs into keratinocytes, which may potentially be applicable for cell transplantation in the clinic and for modeling inherited skin diseases, such as the epidermolysis bullosa (EB) subtypes and congenital ichthyoses (10-12). The protocol has been adapted from our previously published work (13) on the differentiation of mouse iPSCs into keratinocytes as well as studies published by other groups on the differentiation of human ESCs and iPSCs into epithelial and keratinocyte lineages $(14,15)$ with modifications. The resulting iPSC-derived keratinocyte-like cells express the markers specific to authentic basal layer keratinocytes, such as keratin 14 (Krt14) and keratin 5 (Krt5), and are able to reconstitute a normal stratified epidermis when grafted onto an immuno-deficient mouse. The protocol requires the seeding of iPSCs onto Geltrex (Gibco) and collagen type I (ColI) - coated dishes followed by the combined treatment with retinoic acid (RA) to induce iPSC differentiation into an ectodermal fate (16) and with bone morphogenetic protein-4 (BMP4) to block the commitment toward a neural fate (17). In addition, we discovered that growth on collagen type IV (ColIV) - and ColI - coated dishes, which mimics the environment of the basal layer of the skin, improves the efficiency of differentiation to a keratinocyte fate. To enrich for keratinocyte stem cells that are positive for Krt14, a keratin marker confirming commitment of the ectoderm to a keratinocyte fate, we exploit the ability of Krt14 positive cells to rapidly attach to ColI/ColIV-coated surfaces (18).

The methodology for iPSC differentiation toward a keratinocyte lineage relies primarily on the ability to maintain long-term human keratinocyte cultures. Therefore, before initiating this iPSC differentiation protocol, we recommend establishing the growth conditions for culturing normal human keratinocytes that allow for their maintenance in culture for at least 6-10 passages. We found that commercially available CnT-07 medium or EpiLife medium supplemented with EpiLife Defined Growth Supplement (EDGS) promote more efficient expansion of human keratinocytes seeded onto ColI-coated dishes. The growth of differentiated iPSC-derived cultures under keratinocyte cell culture conditions following the rapid attachment to ColI/ColIV-coated plates allows for the efficient enrichment for Krt14 positive keratinocytes up to $80-90 \%(13,15)$.

\section{Materials}

\subsection{Coating Tissue Culture Dishes with Geltrex and Coll}

1. Collagen, type I: $3 \mathrm{mg} / \mathrm{mL}$ solution (Advanced BioMatrix).

2. Geltrex hESC-qualified Reduced Growth Factor Basement Membrane Matrix (Gibco).

3. Dulbecco's Modified Eagle Medium/Nutrient Mixture F-12 (DMEM/F12) (Gibco).

4. $60 \mathrm{~mm}$ tissue culture (TC) dishes. 


\subsection{Plating iPSCs for Differentiation}

1 N2B27 Medium : combine DMEM/F12 and Neurobasal medium (Gibco) in a 1:1 ratio and supplement with $0.1 \mathrm{mM}$ nonessential amino acids, $1 \mathrm{mM}$ glutamine, $55 \mu \mathrm{M}$ 2-mercaptoethanol (2-ME), N2 supplement (100 x) (Life Technologies), B27 supplement (50 x) (Life Technologies), $50 \mu \mathrm{g} / \mathrm{mL}$ ascorbic acid, $0.05 \%$ bovine serum albumin (BSA), $50 \mathrm{U} / \mathrm{mL}$ penicillin-streptomycin, $100 \mathrm{ng} / \mathrm{mL}$ basic FGF (Life Technologies), $10 \mu \mathrm{M}$ Y27632 (Sigma-Aldrich).

3 Dispase (BD).

\subsection{Differentiation of iPSCs with RA and BMP4}

1. $1 \mathrm{mM}$ stock solution of all-trans RA (Sigma-Aldrich) reconstituted in dimethyl sulfoxide (DMSO).

2. $25 \mu \mathrm{g} / \mathrm{mL}$ stock solution of human BMP4 (R\&D Systems) reconstituted in sterile 4 $\mathrm{mM}$ HCL containing $0.1 \%$ BSA.

3. Defined keratinocyte serum-free medium (DKSFM) (Gibco) supplemented with 50 $\mathrm{U} / \mathrm{mL}$ penicillin-streptomycin. DKSFM is sold as a kit containing DKSFM Basal Medium and DKSFM growth supplement.

4. $1 \times$ PBS.

5. CnT-07 epidermal keratinocyte medium (CELLnTEC) containing $50 \mathrm{U} / \mathrm{mL}$ penicillin-streptomycin. CnT-07 is sold as a kit containing CnT Basal Medium and supplements A, B and C.

\subsection{Rapid Attachment and Culturing of iPSC-Derived Keratinocytes}

1. $100 \mathrm{~mm}$ tissue culture dish.

2. Collagen, type IV, powder (Sigma-Aldrich).

3. $0.25 \%$ Glacial acetic acid.

4. Collagen, type I, $3 \mathrm{mg} / \mathrm{ml}$ solution (Advanced BioMatrix).

5. $\mathrm{CnT}-07$ (see 2.3).

6. Accutase (Gibco).

7. $1 \times$ PBS without $\mathrm{Ca}^{2+}$ and $\mathrm{Mg}^{2+}$.

\subsection{Equipment}

1 Biological safety cabinet.

$2 \quad 37^{\circ} \mathrm{C}$ water bath.

$3 \quad 37{ }^{\circ} \mathrm{C} / 5 \% \mathrm{CO}_{2}$ humidified tissue culture incubator.

7 Centrifuge (room temperature). 


\section{Methods}

\subsection{Coating Tissue Culture Dishes with Geltrex and Coll}

The procedure is to be performed in a biological safety cabinet using aseptic techniques. Similar to Matrigel, Geltrex matrix solidifies rapidly at room temperature (RT). Therefore; it is recommended to aliquot each new batch of the matrix upon arrival and use pre-chilled pipet tips, racks and tubes while working with the reagent. We recommend making 50, 100 and $200 \mu \mathrm{L}$ aliquots and to store them at $-80{ }^{\circ} \mathrm{C}$. Use Geltrex at 1:100 dilutions. While the maintenance of feeder-free iPSC cultures requires only Geltrex as a surface coating agent, for iPSC differentiation, the combination of Geltrex and Coll is more efficient to induce the commitment toward a keratinocyte lineage (see Note 1). The coating procedure below is described for a $60 \mathrm{~mm}$ tissue culture dish. If a larger dish is to be used, adjust the volume of the coating solution accordingly.

1. Remove a $50 \mu \mathrm{L}$ aliquot of Geltrex from the $-80{ }^{\circ} \mathrm{C}$ freezer, and place it on ice in the biological safety cabinet.

2. Add $5 \mathrm{~mL}$ of cold sterile $\mathrm{DMEM} / \mathrm{F} 12$ to a $15 \mathrm{~mL}$ conical tube.

3. Use a $1 \mathrm{~mL}$ glass pipet, take $1 \mathrm{~mL}$ cold DMEM/F12 from the $15 \mathrm{~mL}$ conical tube prepared in step 2, and add to the frozen Geltrex. Gently pipet up and down to thaw and dissolve Geltrex. Transfer the dissolved Geltrex to the rest of DMEM/F12 in the $15 \mathrm{~mL}$ conical tube prepared in step 2. Pipet to mix diluted Geltrex.

4. Add $50 \mu \mathrm{L}$ of $3 \mathrm{mg} / \mathrm{mL}$ Coll stock solution into diluted Geltrex from step 3. Pipet to mix diluted geletrex with ColI. Add $4 \mathrm{~mL}$ of coating solution into $60 \mathrm{~mm}$ dish. Tap or swirl the plate to ensure that the entire surface is coated.

5. Incubate the dish with Geltrex/ColI coating solution at $37^{\circ} \mathrm{C}$ in the tissue culture incubator for at least $1 \mathrm{~h}$.

6. Once the coating is complete, leave the coating solution in the dish and proceed with the plating of iPSCs as described in the next subsection (see Section 3.2). Alternatively, aspirate the coating solution and add $2 \mathrm{~mL}$ of fresh DMEM/F12 into the coated dish to prevent it from drying before plating the cells.

\subsection{Plating iPSCs for Differentiation}

Prepare one $60 \mathrm{~mm}$ tissue culture dish of feeder-free iPSCs grown to $\sim 70 \%$ of confluency (see Note 2). Examine cells under a microscope to confirm the absence of contamination and

\footnotetext{
${ }^{1}$ We initially used growth factor reduced BD Matrigel to plate iPSCs for differentiation. However, the combination of Geltrex and ColI gives a higher yield of keratinocytes upon iPSC differentiation.

${ }^{2}$ The provided protocol is optimized for iPSCs generated with an integration-free modified mRNA-based reprogramming approach $(21,22)$. We maintain iPSCs on either mitomycin C inactivated human neonatal fibroblasts or on Geltrex matrix in N2B27 medium (23) under low $\mathrm{O}_{2}$ conditions (5\%). While iPSCs are maintained under low oxygen conditions, the differentiation toward a keratinocyte lineage is performed under atmospheric $\mathrm{O}_{2}(\sim 20 \%)$ in the regular tissue culture incubator. To avoid spontaneous differentiation, the iPSC culture should only be grown to a subconfluent state. Healthy undifferentiated human iPSCs usually form round tight colonies with clear margins (Fig. 2a). Avoid using partially differentiated iPSCs for keratinocyte derivation. Although the provided protocol has been shown to produce functional keratinocytes from human iPSCs generated by an integrating lentivirus approach, there is always a possibility that the partial reactivation of exogenous factors, especially c-Myc and Klf4, may influence the differentiation of these lentivirally-derived iPSCs into keratinocytes, and the protocol may require optimizations for this type of iPSCs.
} 
the maintenance of their undifferentiated phenotype. If the cells are stressed or dying, they start to differentiate, presenting themselves as "cobblestone" areas with larger polymorphic cells, and should not be used for the differentiation toward keratinocytes. For iPSC differentiation toward keratinocytes, we recommend a 1:8 split ratio of iPSCs (see Note 3).

1. Prewarm N2B27 medium and Dispase in the $37^{\circ} \mathrm{C}$ water bath.

2. Using the microscope, confirm that the colonies are ready for passaging. Gently aspirate medium from the dish. Add $2 \mathrm{~mL}$ of $1 \mathrm{x}$ PBS, swirl the plate to wash the cells, and gently aspirate PBS.

3. Add $1 \mathrm{~mL}$ of Dispase and return the plate to the $37^{\circ} \mathrm{C}$ tissue culture incubator for $3-5 \min$.

4. While the cells are being incubated with Dispase, gently aspirate the Geltrex/ColI coating solution (or DMEM/F12) from step 6 in the Geltrex/Coll coating procedure (see Section 3.1) and add $4 \mathrm{~mL}$ of complete N2B27 medium into the coated dish.

5. After 3-5 min incubating with Dispase, confirm that the cells are ready to be picked by looking for rolled or folded edges around the colonies.

6. Transfer the plate to the biological safety cabinet and carefully aspirate Dispase. After the treatment with Dispase, the colonies are very loosely attached to the surface of the dish and may peel off if too much force is used (see Note 4).

7. Gently add $2 \mathrm{~mL}$ of plain DMEM/F12. Aspirate off the medium and repeat the wash 3 times.

8. Add $2 \mathrm{~mL}$ of complete N2B27 into the dish and gently scrape the colonies off the plate. Transfer the cells from the dish into a $15 \mathrm{~mL}$ conical tube and add $6 \mathrm{~mL}$ of complete N2B27 bring the total volume of cell suspension to $8 \mathrm{~mL}$.

9. Gently mix the cell suspension to break large clumps of cells. Transfer $1 \mathrm{~mL}$ of the cell suspension to the coated dish prepared in step 3 of the current subsection.

Discard or replate the leftover cells using the conditions established for a given laboratory (see Note 5).

10. Transfer the newly plated cells to the incubator and gently shake the plate back and forth and side to side to distribute the cells evenly (see Note 6). Incubate the cells overnight in the $37^{\circ} \mathrm{C}$ tissue culture incubator.

\footnotetext{
${ }^{3}$ While we recommend a 1:4 or 1:5 split ratio for the maintenance of iPSCs; for their differentiation, iPSCs need to be seeded as small clumps at very low density to allow for enough surface area for the sufficient expansion of differentiating cells. The colonies should be evenly dispersed in the dish. To achieve this, gently shake the dish from side to side and front to back during passaging. ${ }^{4}$ If iPSC colonies peel off while being incubated with Dispase, collect Dispase with detached iPSC colonies into a $15 \mathrm{~mL}$ conical tube, add plain DMEM/F12 into the dish and gently scrape the remaining colonies off, transfer the colonies from the dish into the $15 \mathrm{~mL}$ conical tube with Dispase and the rest of iPSCs. Spin the cells down at $75 \mathrm{x} g$ for $10 \mathrm{~min}$, aspirate the supernatant. Gently resuspend the iPSC pellet in plain DMEM/F12, spin the cells down at $75 \mathrm{x} g$ for $10 \mathrm{~min}$, repeat the wash 2 times. Proceed with step 9 of Section 3.2.

${ }^{5}$ While we regularly use N2B27 medium for the maintenance of human iPSCs, other media can also be used.

${ }^{6}$ Do not swirl the dish since the cells tend to cluster in the middle when the dish is being swirled.
} 


\subsection{Differentiation of iPSCs with RA and BMP4}

The differentiation and subculturing of iPSC-derived keratinocytes are to be performed in a biological safety cabinet using aseptic techniques. The protocol schematic is outlined in Fig. 1. Examine the new plate the day after passaging to confirm the successful attachment of iPSCs. If iPSCs start forming colonies (Fig. 2a), proceed with the differentiation protocol below (see Note 7).

1. Prewarm complete DKSFM (with antibiotics and DKSFM supplement) in the $37^{\circ} \mathrm{C}$ water bath.

2. Add $5 \mathrm{~mL}$ of prewarmed DKSFM from the previous step to a $15 \mathrm{~mL}$ conical tube, add $5 \mu \mathrm{L}$ of $1 \mathrm{mM}$ RA to achieve $1 \mu \mathrm{M}$ final working concentration and $5 \mu \mathrm{L}$ of 25 $\mu \mathrm{g} / \mathrm{mL}$ BMP4 to achieve $25 \mathrm{ng} / \mathrm{mL}$ final working concentration, mix well.

3. Aspirate off N2B27 medium from the dish with plated iPSCs, wash once with $4 \mathrm{~mL}$ of 1 x PBS, and add $4 \mathrm{~mL}$ of DKSFM containing $1 \mu \mathrm{M}$ RA and $25 \mathrm{ng} / \mathrm{mL}$ BMP4 from the step above. This is day 1 of differentiation procedure.

4. Transfer the cells to the incubator and incubate for $48 \mathrm{~h}$.

5. Replace the medium with fresh DKSFM containing $1 \mu \mathrm{M}$ RA and $25 \mathrm{ng} / \mathrm{mL}$ BMP4 after $48 \mathrm{~h}$ of incubation. Transfer the cell to the incubator for another $48 \mathrm{~h}$.

6. After the second round of $48 \mathrm{~h}$ induction (day 4 of differentiation), replace the medium with complete DKSFM without RA and BMP4. Incubate cells in the incubator for 10 days in complete DKSFM, changing medium every other day.

7. On day 14 of differentiation, prepare complete CnT-07 medium by adding antibiotics and provided supplements, pre-warm the medium. By this day, the majority of the cells in the outgrown iPSC colony start exhibiting an epithelial-like phenotype (see Fig. 2b).

8. Aspirate off DKSFM from differentiated cells, and replace with $4 \mathrm{~mL}$ of complete CnT-07. Incubate the cells in the tissue culture incubator for another 10 days, changing complete CnT-07 every other day.

\subsection{Rapid Attachment and Culturing of iPSC-Derived Keratinocytes}

On day 24 of differentiation, many cells that migrate away from the outgrown iPSC colony will exhibit a keratinocyte-like phenotype (Fig. 2c), and start expressing p63, a master regulator required for the commitment of the ectoderm to a keratinocyte fate $(19,20)$, and Krt14 (see Note 8). By this day, the $60 \mathrm{~mm}$ dish used for iPSC differentiation is fully confluent and need to be passaged. To enrich for iPSC-derive keratinocytes during passaging, we perform the rapid attachment of the differentiated iPSC culture to ColI/ColIVcoated plates. We recommend using up to four $100 \mathrm{~mm}$ ColI/ColIV-coated tissue culture dishes to perform the rapid attachment procedure from one $60 \mathrm{~mm}$ dish containing

\footnotetext{
${ }^{7}$ If the colonies start to differentiate spontaneously, discard the dish and repeat the replating of iPSCs using a fresh iPSC culture. ${ }^{8}$ We are able to obtain a maximum of $25-30 \%$ of K14 positive cells in the entire culture before the rapid attachment step. The efficiency of differentiation usually varies from $5 \%$ to $30 \%$ among experiments and among iPSC lines.
} 
differentiated iPSCs. If only one $100 \mathrm{~mm}$ dish is to be used, plate one fourth of the differentiated iPSC culture for the rapid attachment procedure.

3.4.1 Coating Plates with Coll and CollV-The procedure is to be performed in the biological safety cabinet using aseptic techniques.

1. Reconstitute ColIV powder to a concentration of $2 \mathrm{mg} / \mathrm{mL}$ in sterile $0.25 \%$ Glacial acetic acid. Dissolve for several $\mathrm{h}$ at $2-8^{\circ} \mathrm{C}$, occasionally swirling. Make aliquots and store them at $-20{ }^{\circ} \mathrm{C}$.

2. Thaw the aliquot of ColIV stock solution $(2 \mathrm{mg} / \mathrm{mL})$ very slowly by placing the vial in an ice bucket and keeping it at $4^{\circ} \mathrm{C}$ for several $\mathrm{h}$.

3. Resuspend ColIV stock solution in the appropriate volume $(5 \mathrm{~mL}$ per each $100 \mathrm{~mm}$ dish) of sterile $0.25 \%$ Glacial acetic acid to a final working concentration of 7 $\mu \mathrm{g} / \mathrm{mL}$. Add an appropriate volume of ColI stock solution to achieve a final working Coll concentration of $30 \mu \mathrm{g} / \mathrm{mL}$. Coat the plates by using $5 \mathrm{~mL}$ of working solution to cover a $100 \mathrm{~mm}$ dish. Incubate the plates at room temperature in the biological safety cabinet for $1 \mathrm{~h}$.

4. Aspirate the liquid from the coated plates, rinse the dishes once with $5 \mathrm{~mL}$ of sterile $1 \mathrm{x}$ PBS and once with $5 \mathrm{~mL}$ of $\mathrm{ddH}_{2} \mathrm{O}$.

5. Air-dry the washed dishes in the biological safety cabinet. Use plates directly or seal them with Parafilm and store at $4^{\circ}$ for up to 6 months. To use a previously stored ColIV-coated plate, allow the plate to warm up at room temperature in the biological safety cabinet for at least $1 \mathrm{~h}$ prior to plating cells.

\subsubsection{Rapid attachment of iPSC-derived keratinocytes}

1. On day 24 of differentiation, prewarm complete CnT-07, Accutase, and ColI/ ColIV-coated dish(es).

2. Wash the cells with $1 \times$ PBS, add $2 \mathrm{~mL}$ of Accutase and incubate in the tissue culture incubator for $5 \mathrm{~min}$ (see Note 9). Confirm under the microscope that cells start detaching.

3. Add $3 \mathrm{~mL}$ of complete Cnt-07, pipet up and down to dislodge the cells and collect the cell suspension into a $15 \mathrm{~mL}$ conical tube. Spin the cells down at $260 \mathrm{x} g$ for 5 min and aspirate the supernatant. Resuspend the pellet in $10 \mathrm{~mL}$ of complete Cnt-07 medium, repeat the spin at $260 \times \mathrm{g}$ for $5 \mathrm{~min}$, and aspirate the supernatant.

4. Resuspend the pellet in $4 \mathrm{~mL}$ of complete CnT-07, pipet up and down to break cell clumps into single cells.

5. Add $9 \mathrm{~mL}$ of complete CnT-07 medium into each ColI/ColIV-coated dish and transfer $1 \mathrm{~mL}$ of cell suspension from step 4 above into each ColI/ColIV-coated dish. Allow the cells to attach to the coated dish at room temperature for 15-30 min (see Note 10).

\footnotetext{
${ }^{9} \mathrm{We}$ do not recommend using trypsin at this stage of the protocol.
} 
6. Carefully aspirate the medium with the floating cells (these are undifferentiated or partially differentiated iPSCs). Do not disturb the attached cells (these are iPSCderived Krt14 positive cells). Add $10 \mathrm{~mL}$ of fresh complete CnT-07 medium into the plate with the attached cells. Let the cells expand in the $37^{\circ} \mathrm{C}$ tissue culture incubator, changing the medium every other day. Passage cells as needed (see Note 11) with Accutase in CnT-07 or EpiLife (with EDGS supplement) on ColI-coated dishes. After passage 2 or 3 and following the rapid attachment step, the culture should consist of $\sim 90 \%$ of Krt14 positive cells exhibiting a keratinocyte-like phenotype (see Fig. 2d). The keratinocyte-like phenotype of the obtained culture can be verified by standard immunflorescence analyses for Krt14 expression and by the ability to reconstitute a normal stratified epidermis in organotypic cultures.

\section{Acknowledgments}

We are grateful for funding support from the National Institutes of Health (R01AR059947 and P30 AR057212), the US Department of Defense (PR110793), the Foundation for Ichthyosis \& Related Skin Types (F.I.R.S.T.) and the Dystrophic Epidermolysis Bullosa Research Association (DEBRA) International.

\section{Abbreviations}

$\begin{array}{ll}\text { ColI } & \text { type I collagen } \\ \text { ColIV } & \text { type IV collagen } \\ \text { ESC } & \text { embryonic stem cell } \\ \text { iPSC } & \text { induced pluripotent stem cell } \\ \text { Krt14 } & \text { keratin } 14\end{array}$

RA retinoic acid, BMP4, bone morphogenetic protein-4

\section{References}

1. Takahashi K, Yamanaka S. Induction of pluripotent stem cells from mouse embryonic and adult fibroblast cultures by defined factors. Cell. 2006; 126(4):663-76. [PubMed: 16904174]

2. Takahashi K, Tanabe K, Ohnuki M, Narita M, Ichisaka T, Tomoda K, Yamanaka S. Induction of pluripotent stem cells from adult human fibroblasts by defined factors. Cell. 2007; 131(5):861-72. [PubMed: 18035408]

3. Yu J, Vodyanik MA, Smuga-Otto K, Antosiewicz-Bourget J, Frane JL, Tian S, Nie J, Jonsdottir GA, Ruotti V, Stewart R, Slukvin II, Thomson JA. Induced pluripotent stem cell lines derived from human somatic cells. Science. 2007; 318(5858):1917-20. [PubMed: 18029452]

4. Okita K, Ichisaka T, Yamanaka S. Generation of germline-competent induced pluripotent stem cells. Nature. 2007; 448(7151):313-7. [PubMed: 17554338]

5. Wernig M, Meissner A, Foreman R, Brambrink T, Ku M, Hochedlinger K, Bernstein BE, Jaenisch R. In vitro reprogramming of fibroblasts into a pluripotent ES-cell-like state. Nature. 2007; 448(7151):318-24. [PubMed: 17554336]

\footnotetext{
${ }^{10}$ If only a few cells attach, incubate the plate for up to an hour in the $37^{\circ} \mathrm{C}$ tissue culture incubator. Alternatively, skip the rapid attachment during the first passage. Instead, split the entire plate of differentiated iPSCs onto four fresh ColI-coated dishes in complete CnT-07. Let the cells reach 60-70\% confluency and then perform the rapid attachment plating as described in Section 3.4.

${ }^{11}$ It may take up to 2 weeks to expand the culture of iPSC-derived keratinocytes post- rapid attachment plating. Do not allow the cells to overgrow, since this will induce premature differentiation. Ideally, the cells should be subcultured onto a fresh ColI-coated dish once they reach $60 \%$ confluency. We recommend using Accutase instead of trypsin for keratinocyte passaging.
} 
6. Yamanaka S. Strategies and new developments in the generation of patient-specific pluripotent stem cells. Cell Stem Cell. 2007; 1(1):39-49. [PubMed: 18371333]

7. Nishikawa S, Goldstein RA, Nierras CR. The promise of human induced pluripotent stem cells for research and therapy. Nat Rev Mol Cell Biol. 2008; 9(9):725-9. [PubMed: 18698329]

8. Yamanaka S. A fresh look at iPS cells. Cell. 2009; 137(1):13-7. [PubMed: 19345179]

9. Takahashi K. Cellular reprogramming--lowering gravity on Waddington's epigenetic landscape. J Cell Sci. 2012; 125(Pt 11):2553-60. [PubMed: 22736045]

10. Khavari PA. Therapeutic gene delivery to the skin. Mol Med Today. 1997; 3(12):533-8. [PubMed: 9449124]

11. DiGiovanna JJ, Robinson-Bostom L. Ichthyosis: etiology, diagnosis, and management. Am J Clin Dermatol. 2003; 4(2):81-95. [PubMed: 12553849]

12. Smith F. The molecular genetics of keratin disorders. Am J Clin Dermatol. 2003; 4(5):347-64. [PubMed: 12688839]

13. Bilousova G, Chen J, Roop DR. Differentiation of mouse induced pluripotent stem cells into a multipotent keratinocyte lineage. J Invest Dermatol. 2011; 131(4):857-64. [PubMed: 21150926]

14. Metallo CM, Ji L, de Pablo JJ, Palecek SP. Retinoic acid and bone morphogenetic protein signaling synergize to efficiently direct epithelial differentiation of human embryonic stem cells. Stem Cells. 2008; 26(2):372-80. [PubMed: 17962700]

15. Itoh M, Kiuru M, Cairo MS, Christiano AM. Generation of keratinocytes from normal and recessive dystrophic epidermolysis bullosa-induced pluripotent stem cells. Proc Natl Acad Sci U S A. 2011; 108(21):8797-802. [PubMed: 21555586]

16. Bain G, Kitchens D, Yao M, Huettner JE, Gottlieb DI. Embryonic stem cells express neuronal properties in vitro. Dev Biol. 1995; 168(2):342-57. [PubMed: 7729574]

17. Gambaro K, Aberdam E, Virolle T, Aberdam D, Rouleau M. BMP-4 induces a Smad-dependent apoptotic cell death of mouse embryonic stem cell-derived neural precursors. Cell Death Differ. 2006; 13(7):1075-87. [PubMed: 16311513]

18. Bickenbach JR, Chism E. Selection and extended growth of murine epidermal stem cells in culture. Exp Cell Res. 1998; 244(1):184-95. [PubMed: 9770361]

19. Mills AA, Zheng B, Wang XJ, Vogel H, Roop DR, Bradley A. p63 is a p53 homologue required for limb and epidermal morphogenesis. Nature. 1999; 398(6729):708-13. [PubMed: 10227293]

20. Koster MI, Kim S, Mills AA, DeMayo FJ, Roop DR. p63 is the molecular switch for initiation of an epithelial stratification program. Genes Dev. 2004; 18(2):126-31. [PubMed: 14729569]

21. Warren L, Manos PD, Ahfeldt T, Loh YH, Li H, Lau F, Ebina W, Mandal PK, Smith ZD, Meissner A, Daley GQ, Brack AS, Collins JJ, Cowan C, Schlaeger TM, Rossi DJ. Highly efficient reprogramming to pluripotency and directed differentiation of human cells with synthetic modified mRNA. Cell Stem Cell. 2010; 7(5):618-30. [PubMed: 20888316]

22. Warren L, Ni Y, Wang J, Guo X. Feeder-free derivation of human induced pluripotent stem cells with messenger RNA. Sci Rep. 2012; 2:657. [PubMed: 22984641]

23. Liu Y, Song Z, Zhao Y, Qin H, Cai J, Zhang H, Yu T, Jiang S, Wang G, Ding M, Deng H. A novel chemical-defined medium with bFGF and N2B27 supplements supports undifferentiated growth in human embryonic stem cells. Biochem Biophys Res Commun. 2006; 346(1):131-9. [PubMed: 16753134] 


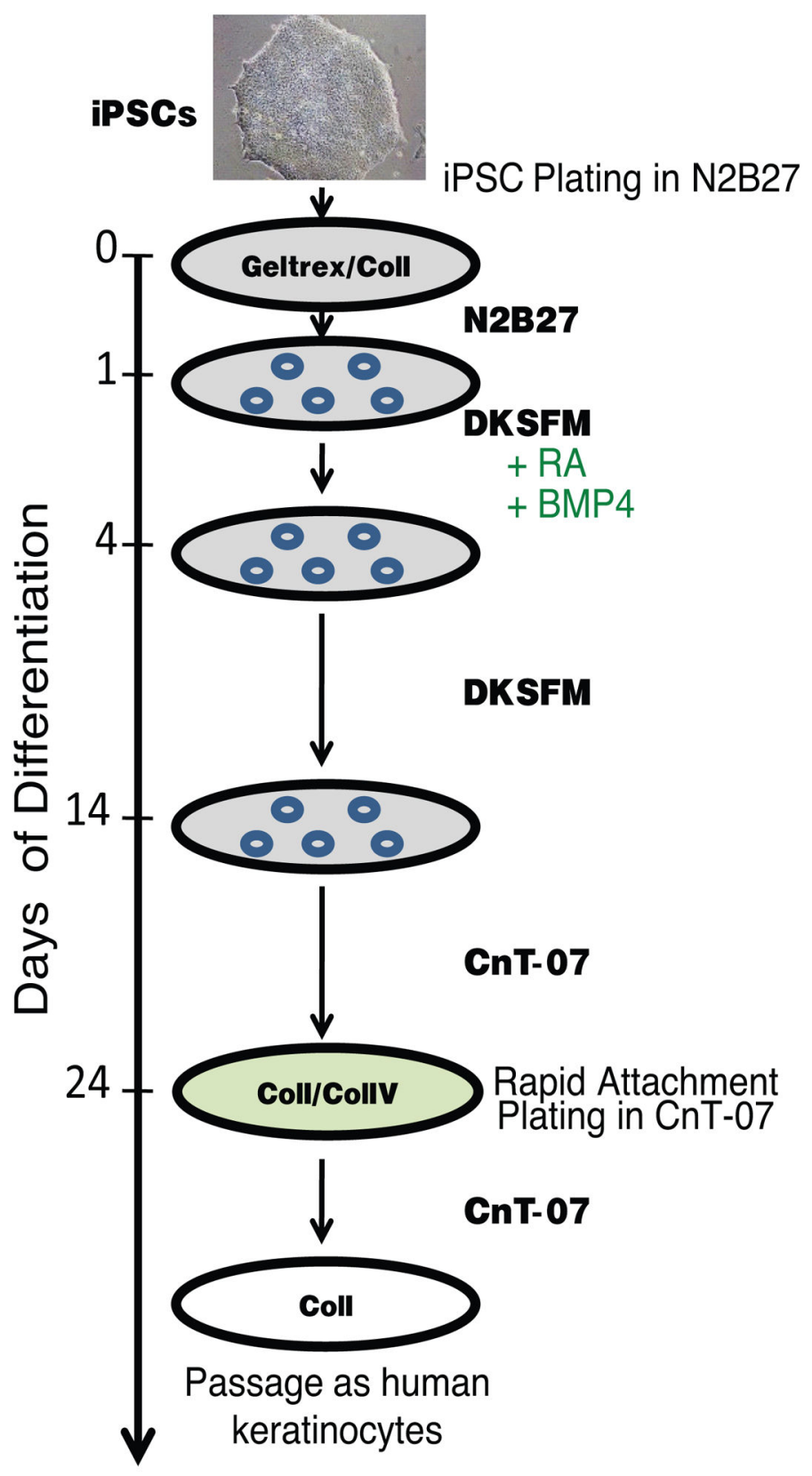

Fig. 1.

Schematic representation of the protocol for the differentiation of human iPSCs into a keratinocyte lineage. 

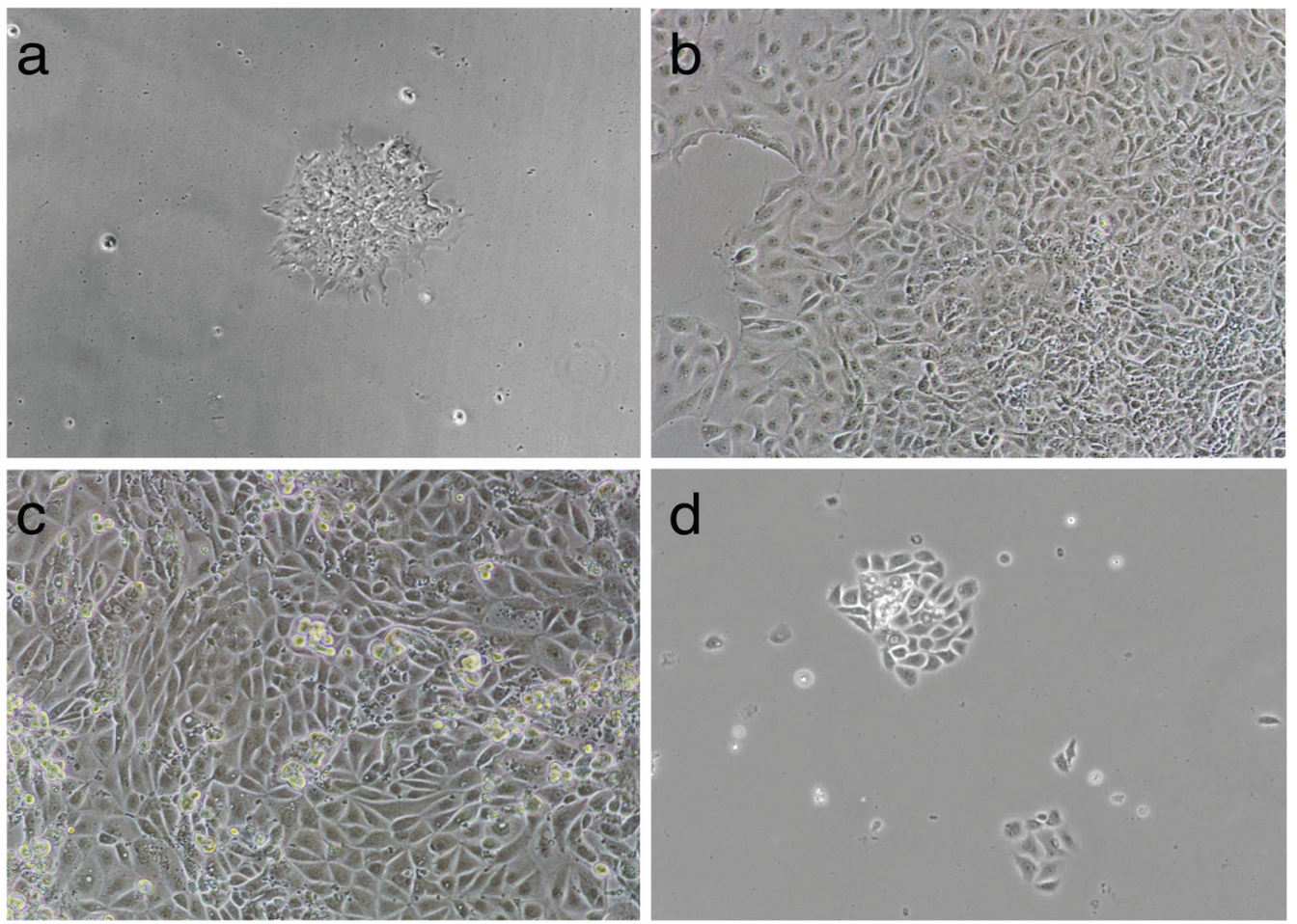

Fig. 2. The stages of iPSC differentiation during keratinocyte derivation

Human iPSCs generated with a modified mRNA-based approach from human neonatal fibroblasts were differentiated into keratinocytes using RA and BMP4. (a) Human iPSCs seeded at low density for differentiation on day 1 of differentiation. (b) An iPSC colony outgrown on a Geltrex/ColI-coated plate after the combined treatment with RA and BMP4 on day 14 of differentiation. (c) An iPSC colony outgrown on a Geltrex/ColI-coated plate on day 24 of differentiation before rapid attachment plating. (d) iPSC-derived keratinocytes at passage 1 post-rapid attachment plating (day 29 of differentiation, day 5 post-rapid attachment). All images were taken with 10x objectives. 